\title{
Restrição de crescimento intrauterino
}

\author{
Intrauterine growth retardation
}

\section{Restricción de crecimiento intrauterino}

\author{
Dailson D. S. Pereira, ${ }^{*}$ Alessandra L. C. Magalhães, Nilson R. de Jesús, Alexandre J. B. Trajano
}

\section{Resumo}

São denominados fetos pequenos para a idade gestacional (PIG) aqueles que têm o peso estimado abaixo do percentil 10. A Restrição do Crescimento Intrauterino (CIR) é a principal causa de fetos PIG e está associada à alta taxa de morbimortalidade fetal e neonatal. Seu adequado diagnóstico, além do acompanhamento, tem impacto como marcador de assistência à mãe e ao feto. Esta revisão tem como objetivo auxiliar o manejo desta grave condição, tentando auxiliar na diminuição dos efeitos deletérios sobre o feto e o neonato. Atualmente, além do investimento em tratamento neonatal, tem-se procurado investir no reconhecimento precoce das pacientes com maior risco de terem fetos com CIR e nas formas terapêuticas de aumentar sua prevenção, diminuindo, assim, sua incidência e gravidade. As principais causas de CIR são: alterações genéticas, infecções e insuficiência placentária. Reconhecer estas causas ajuda na decisão do momento apropriado do parto. De todas, a insuficiência placentária é a mais comum e a que apresenta os maiores desafios, por ser paradoxalmente a que tem menor potencial letal e, ao mesmo tempo, a que mais mata em nosso meio. O adequado manejo de gestações com CIR causado pela insuficiência placentária impõe a necessidade do pleno conhecimento da sequência das alterações hemodinâmicas feto-placentárias ao Doppler, para a decisão em relação a necessidade e o momento adequado, dependendo da idade gestacional (IG), de se antecipar o parto, tendo em vista a ação danosa que a prematuridade pode somar a estes fetos, com repercussões desfavoráveis que podem ir além do período perinatal.

Descritores: Retardo do Crescimento Fetal, RCIU; Ultrassonografia doppler; Insuficiência placentária.

\begin{abstract}
Are called ferns small for gestational age (SGA) those who have the estimated weight percentile below 10. Intrauterine growth restriction (IUGR) is the leading cause of SGA fetuses and is associated with high rate of fetal and neonatal morbidity and mortality. Proper diagnosis and monitoring has an impact as a marker of assistance for the mother and the fetus. This review aims to assist the management of this serious condition, trying to assist in reducing the deleterious effects on the fetus and the neonate. Currently, in addition to the investment in neonatal treatment, has sought to invest in the early recognition of patients with higher risk of having fetuses with IUGR and in therapeutic ways to increase its prevention, thus decreasing its incidence and severity. The main causes of IUGR are: genetic changes, infections and placental insufficiency. Recognize these causes help in the decision of the appropriate time of childbirth. Of all, the placental insufficiency is the most common and which presents the greatest challenges because it is paradoxically the least lethal potential and at the same time the most kills in our midst. The appropriate management of pregnancies with IUGR caused by placental insufficiency imposes the necessity of the full knowledge of the sequence of the hemodynamic changes fetus-placental Doppler to, for the decision about the necessity and the appropriate time, depending on the gestational age (GA), to anticipate the birth with a view to damaging action that prematurity can sum these ferns, with unfavourable repercussions that may go beyond the perinatal period.
\end{abstract}

Keywords: Intrauterine Growth Retardation, IUGR; Ultrasonography, doppler; Placental insufficiency.

Revista HUPE, Rio de Janeiro, 2014;13(3):32-39

Recebido: 24/04/2014 | Revisado: 02/07/2014 | Aprovado: 28/07/2014

doi: 10.12957/rhupe.2014.12135
"Endereço para correspondência: Av. Professor Manoel de Abreu, 500, 10 andar, Rio de Janeiro, RJ, Brasil. CEP: 20550-170.

E-mail: dailsondamian@globo.com 


\section{Resumen}

Son denominados fetos pequeños para la edad gestacional (PEG) aquellos que tienen el peso estimado bajo el percentil 10. La restricción del crecimiento intrauterino (RCIU) es la principal causa de fetos PEG y está asociada a la alta tasa de morbimortalidad fetal y neonatal. Su adecuado diagnóstico y acompañamiento tienen impacto como marcador de asistencia a la madre y al feto. Esta revisión tiene como objetivo ayudar en el manejo de esta grave condición, intentando disminuir los efectos deletéreos sobre el feto y el neonato. Actualmente, más allá de la inversión en tratamiento neonatal, se ha buscado invertir en el reconocimiento precoz de las pacientes con mayor riesgo de tener fetos con RCIU y en las formas terapéuticas de aumentar la prevención, disminuyendo, así, la incidencia y gravedad. Las principales causas de RCIU son: alteraciones genéticas, infecciones e insuficiencia placentaria. Reconocer estas causas ayuda en la decisión del momento adecuado para el parto. De todas, la insuficiencia placentaria es la más común y la que presenta los mayores desafíos, por ser paradójicamente la que tiene menor potencial letal y al mismo tiempo la que más mata en nuestro medio. El adecuado manejo de gestaciones con RCIU causado por la insuficiencia placentaria exige la necesidad del pleno conocimiento de la secuencia de las alteraciones hemodinámicas feto-placentarias al Doppler, para la decisión respecto a la necesidad y el momento adecuado, dependiendo de la edad gestacional (EG), de anticiparse al parto, teniendo en cuenta la acción dañina que la prematuridad puede sumar a estos fetos, con repercusiones desfavorables que pueden ir más allá del periodo perinatal.

Palabras clave: Retardo del Crescimiento Intrauterino, RCIU; Ultrasonografía doppler; Insuficiencia placentaria.

\section{Introdução}

Restrição de crescimento intrauterino (CIR) é associada com morbidade e mortalidade perinatal aumentada, ${ }^{1}$ assim como com maior morbidade em longo prazo e predisposição a doenças crônicas na idade adulta. ${ }^{2}$ Apesar das opções limitadas de tratamento intra útero, o diagnóstico pré-natal é essencial já que foi observado que fetos com restrição de crescimento não diagnosticados no período pré-natal apresentavam um risco quatro vezes maior de desfechos adversos quando comparados com aqueles diagnosticados durante a gestação. ${ }^{3}$ Portanto, a vigilância do bem-estar fetal é o ponto chave do manejo obstétrico, permitindo a programação do momento ideal do parto.

\section{Definição}

O feto PIG é definido como aquele com um peso estimado pela ultrassonografia abaixo do percentil 10 para idade gestacional. ${ }^{4}$ Esta definição acarreta na inclusão em um grupo de risco de fetos que não apresentarão nenhum dano futuro. Alguns autores recomendam que a classificação mais correta deveria incluir medidas seriadas da biometria fetal. ${ }^{5}$ Quaisquer que sejam os parâmetros de classificação usados, é fundamental a correta datação da idade gestacional, a respeito da data da última menstruação de certeza (DUM) e pela ecografia obstétrica precoce, preferencialmente pela medida do comprimento cabeçanádega $(\mathrm{CCN}){ }^{6}$
Pode haver fetos PIGs sem que haja a presença de patologias. São ditos pequenos constitucionais e cursam com baixo risco de hipóxia fetal. Geralmente, sua curva de crescimento, embora abaixo do p10, é paralela a curva normal.

No entanto, também há situações como a pré-eclâmpsia, infecções, doença do anticorpo antifosfolipídeo e aneuploidias que vão causar a CIR e aumentam o risco de hipóxia fetal.

É importante a diferenciação entre a CIR de início precoce e aquela de início tardio, que têm diferentes características bioquímicas, biofísicas e clínicas. Na primeira forma, ocorrida na fase de hiperplasia celular fetal, a restrição de crescimento fetal é simétrica e mais comumente associada ao uso de drogas, alterações genéticas e infecções fetais, principalmente pelo TORCHS. ${ }^{7}$ Neste tipo, é comum o reconhecimento de alterações estruturais do feto à ecografia. Outro achado ecográfico que sugere este tipo é a presença de polidramnia junto com o feto simetricamente menor. ${ }^{5}$ Vale ressaltar que a maioria dos fetos simetricamente pequenos são constitucionais e não apresentarão alterações estruturais ou risco de dano fetal. ${ }^{5}$ Depois de instalada a CIR nesta fase, a letalidade é alta e possibilidade de manejo médico é pequena.

CIR tardio ocorre na fase de hipertrofia celular fetal e sua principal causa é a insuficiência placentária, estando, portanto, ligada a préeclâmpsia e sendo caracterizado por uma assimetria do crescimento fetal, com a circunferência abdominal menor que o esperado para a IG 
e menor proporcionalmente que o perímetro cefálico e as medidas de ossos longos. É neste tipo de CIR que o médico pode intervir para diminuir a morbimortalidade. Geralmente, não há malformações fetais. É comum o aparecimento de oligodramnia, principalmente em casos mais graves. Outros achados comuns são alterações hemodinâmicas fetais, mais facilmente reconhecidas por meio da dopplerfluxometria. ${ }^{5} \mathrm{Na}$ maioria dos casos, a CIR é secundária à insuficiência placentária. $^{8}$

O tipo misto ocorre na fase concomitante de hiperplasia e hipertrofia celular, entre 16 e 32 semanas de gravidez.

Vale ressaltar que também podemos encontrar o início da instalação da insuficiência placentária de forma precoce, na fase de hiperplasia celular, levando a CIR simétrico. Geralmente, o desfecho é pior nesta condição.

\section{Rastreio/diagnóstico}

Anamnese: a identificação de fatores de risco para CIR deve ser realizada rotineiramente na $1^{\text {a }}$ consulta pré-natal. Dentre eles, podemos citar:

- História pregressa de CIR ou de natimorto: mulheres que tiveram um feto com CIR em gestação prévia têm risco de recorrência de $50 \%{ }^{9}$ Além disso, mais da metade dos óbitos fetais intrauterinos de fetos morfologicamente normais está associado com CIR, principalmente se ocorrido antes de 32 semanas de gravidez. ${ }^{10}$

- Diabetes: apresentam risco elevado de $\mathrm{CIR}^{8}$ assim como importante associação com pré-eclâmpsia, podendo chegar a $50 \%$ tal associação.

- Obesidade: aumenta o risco de fetos pequenos para idade gestacional em 50\%."

- Gestação múltipla: a CIR é responsável por grande parcela da maior taxa de morbidade e mortalidade na gestação múltipla. ${ }^{2}$

- Pré-eclâmpsia: pode causar CIR e é um indicador de sua severidade, especialmente quando iniciada antes de 37 semanas. ${ }^{13}$

- Síndrome do anticorpo antifosfolipídeo: a fisiopatologia da CIR envolve a trombose

\section{placentária. ${ }^{14}$}

Outras causas que podem ser citadas são: infecções maternas e congênitas, uso de drogas, tabagismo e etilismo, desnutrição materna, doenças renais, doenças do colágeno, malformações fetais, cromossomopatias, anomalias placentárias e do cordão umbilical. ${ }^{14}$

Marcadores bioquímicos: diversos marcadores bioquímicos vêm sendo pesquisados para o rastreio de pacientes com maior risco de desenvolvimento de CIR. Dentre estes, podemos citar a proteína plasmática A (PAPP-A) e a fração livre do hCG que, quando em níveis baixos no primeiro trimestre, têm associação com CIR e pré-eclâmpsia. ${ }^{15,16}$ No segundo trimestre, temos a elevação da alfa fetoproteína sérica ou da inibina A como marcadores de risco. ${ }^{17-19}$

Artérias uterinas: a avaliação das artérias uterinas foi proposta como método de rastreio com taxas de detecção variando de 25 a 75\% de acordo com a taxa de falso positivo adotada. ${ }^{20}$ Estratégias de utilização de fatores de risco maternos, pressão arterial, marcadores bioquímicos e Doppler de artérias uterinas no primeiro trimestre vêm sendo pesquisadas para rastreio de CIR e pré-eclâmpsia. ${ }^{21}$

Medida do fundo uterino: a medida da altura do fundo uterino (AFU) é mais um método de vigilância do que de rastreio visto que sua força recai na medida seriada. ${ }^{8}$ Tal vigilância é recomendada pelo Royal College of Obstetrics and Gynaecologists. ${ }^{22}$ Indicações para investigação adicional incluem medidas abaixo do percentil 10 para idade gestacional e medidas seriadas mostrando crescimento abaixo do esperado. ${ }^{8}$

Ultrassonografia de segundo e terceiro trimestres: a efetividade da avaliação biométrica por esta ultrassonografia para rastreio de CIR e seu impacto no desfecho perinatal em gestações de baixo risco é incerto.

Por outro lado, em gestações de alto risco, a avaliação fetal através da estimativa de peso ou da medida da circunferência abdominal é o melhor preditor do crescimento fetal, ${ }^{23}$ sendo a avaliação seriada da biometria fetal o padrão-ouro para o rastreio de CIR. ${ }^{22}$ Estimativas seriadas devem ser realizadas em prazo não menor que duas semanas visando evitar a margem de erro inerente ao método. ${ }^{6}$ 


\section{Manejo}

Como há pouco a se fazer no tratamento intraútero das outras causas de CIR, iremos nos ater ao manejo da insuficiência placentária como causa.

Doppler fetal (artéria umbilical/artéria cerebral média-ACM): os índices de resistência das artérias umbilicais refletem a resistência ao fluxo do território placentário e se correlaciona com a condição fetal e o desfecho da gravidez. ${ }^{24}$ A aplicação da dopplerfluxometria nas gestações complicadas com CIR levou à diminuição da taxa de mortalidade perinatal e admissão na UTI neonatal. ${ }^{25}$

A progressão fisiopatológica deste vaso inicia-se com o aumento de sua resistência, passando pela centralização fetal e podendo chegar à diástole zero e diástole reversa. Centralização fetal consiste na redistribuição do fluxo fetal em situações de hipóxia fetal, priorizando o fluxo para órgãos nobres: cérebro, coração e adrenais. ${ }^{26}$ Seu diagnóstico é dado quando a resistência das artérias umbilicais, em constante elevação nesta patologia, ultrapassa a resistência das ACMs, em queda pela redistribuição. ${ }^{26}$

O fluxo diastólico ausente ou reverso é mais comumente encontrado na CIR de início precoce e é relatado que esses padrões se apresentam em média uma semana antes da deterioração fetal. ${ }^{27}$ Estudos recentes sugerem que esse achado tem valor na predição da morbidade e mortalidade perinatal independente da idade gestacional. ${ }^{28-29}$

Líquido amniótico: estudos longitudinais em CIR por insuficiência placentária de início precoce mostraram que o volume de líquido decresce progressivamente. ${ }^{30}$ Em contrapartida, fetos com CIR com aneuploidias ou malformações apresentam polidramnia.

Ducto venoso: com a deterioração fetal, a pressão venosa central aumenta e as velocidades diastólicas no ducto venoso caem progressivamente levando à onda $\mathrm{A}$ (que reflete a contração atrial no final da diástole) em direção à linha de base e tornado-a ausente/negativa. Estas alterações são tardias e indicam falência cardíaca fetal iminente. ${ }^{27-29} \mathrm{O}$ ducto venoso está associado à acidose fetal. A onda A ausente ou reversa está associada com mortalidade perinatal independente da idade gestacional no momento do parto. ${ }^{28,29}$
Cardiotocografia: é falha ao demonstrar efeito na redução da mortalidade perinatal. A análise da microscilação (short term variation) através da cardiotocografia computadorizada, no entanto, se correlaciona bem com acidose fetal e hipóxia severa e se torna anormal coincidentemente com o ducto venoso. ${ }^{30}$

Perfil biofísico fetal: a alta taxa de falso positivo (50\%) e a ausência de demonstração de benefícios nas gestações de alto risco limitam a sua utilidade clínica. Além disso, as alterações no tônus, movimentos respiratórios e movimentos corporais são muito tardias, em média de 48 a 72 horas após o ducto venoso. ${ }^{28,29}$

\section{Definição do parto}

Conforme já mencionado, as possibilidades de tratamento intraútero são limitadas. Logo, o manejo obstétrico visa o parto de um feto a tempo de evitar lesão hipóxica e morte intrauterina balanceado contra a morbidade potencial causada pela prematuridade. Em alguns casos, o manejo será ditado pelas condições maternas já que a CIR é frequentemente associada a doenças maternas com lúpus eritematoso, pré-eclâmpsia e nefropatia.

Quando a CIR é associada à cromossomopatias e malformações fetais, o grau de letalidade e morbidade relacionado também deve ser levado em consideração no momento da decisão, geralmente ocorrendo no termo, para que não se agregue a prematuridade aos riscos fetais já existentes.

Com relação à via de parto, vale lembrar que se uma hipóxia fetal relativa está presente antes do início do trabalho de parto, mesmo contrações uterinas normais podem ocasionar um quadro de sofrimento fetal agudo. ${ }^{6}$ Assim, a cesariana é mais frequente em pacientes com restrição do crescimento fetal, principalmente quando há alteração na dopplerfluxometria. O feto com CIR em trabalho de parto, induzido ou espontâneo, deve ser continuamente monitorado já que apresenta maior risco de sofrimento agudo. ${ }^{6}$

Para definição do momento do parto é importante o conhecimento adequado do padrão de deterioração clínica destes fetos, o que é determinado pela idade gestacional de início do quadro de restrição do crescimento. Na CIR por insuficiência 


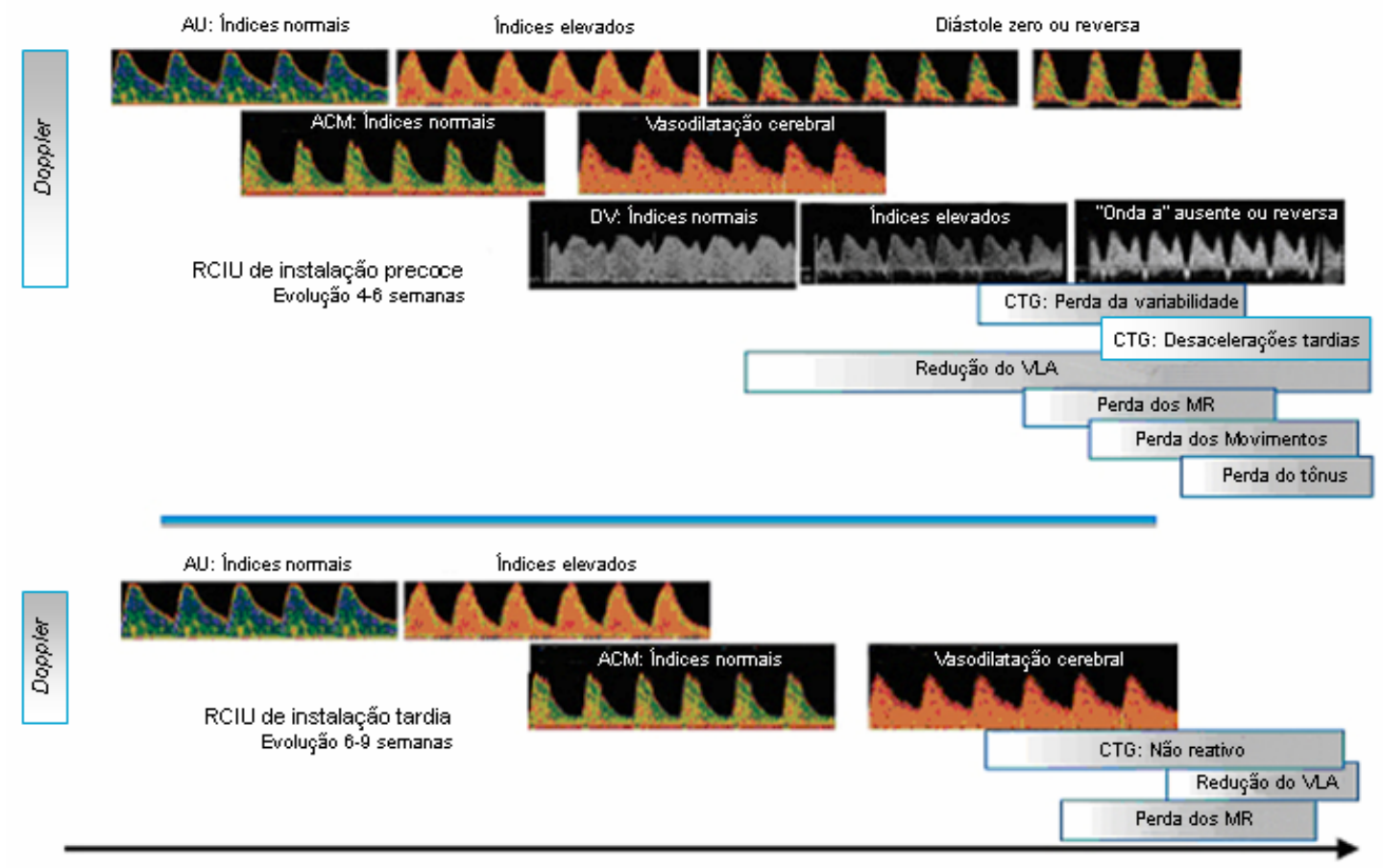

Figura 1. Comparação entre a evolução do comprometimento fetal de instalação precoce e tardia. A figura sumariza a evolução temporal das alterações biofísicas observadas na dopplervelocimetria, volume do líquido amniótico, na cardiotocografia e no perfil biofísico fetal. Os "índices" se referem aos "Índices de Resistência e de Pulsatilidade" da dopplervelocimetria. AU, artéria umbilical; ACM, artéria cerebral média; CTG, cardiotocografia; VLA, volume de líquido amniótico; MR, movimento respiratório. Adaptado de BASCHAT, Ultrasound Obstet Gynecol, 2011.

placentária diagnosticada antes de 34 semanas ocorre aumento progressivo da resistência na artéria umbilical, acompanhada pela centralização fetal, diminuição progressiva do fluxo umbilical diastólico até estar ausente e seguida pela deterioração do ducto venoso, da cardiotocografia e do perfil biofísico fetal. ${ }^{29}$ Tal progressão ocorre em média em 4 a 6 semanas, e esta velocidade é ditada pela rapidez com que o fluxo diastólico na artéria umbilical se torna ausente/reverso. ${ }^{29} \mathrm{Na}$ CIR de início tardio, por sua vez, a resistência da artéria umbilical é pouco aumentada ou mesmo normal, podendo ocorrer a dilatação da artéria cerebral média de forma isolada. ${ }^{29}$ Tal sequência de eventos é ilustrada na figura 1.

Na decisão do momento do parto tem papel fundamental a idade gestacional do feto visto que quanto maior a idade gestacional menor o impacto da prematuridade e, portanto maior a influência de parâmetros biofísicos no prognóstico perinatal e na morbidade de longo prazo. ${ }^{28,29}$ Vale ressaltar a importância de uma equipe neonatal preparada e o conhecimento do ponto de corte para idade gestacional na UTI neonatal. No caso do Núcleo Perinatal do Hospital Universitário Pedro Ernesto (NU-HUPE) este ponto de corte é de 27 semanas, motivo pelo qual não indicamos a antecipação do parto antes desta idade gestacional. Segundo Baschat e colaboradores, ${ }^{28}$ antes de 27 semanas o peso da prematuridade é tão grande que mesmo o ducto venoso sozinho não fornece suficiente estratificação de risco.

Entre 27 e 30 semanas, a conduta no NU-HUPE é ditada pelo ducto venoso, pois nesta idade gestacional cada dia do feto in utero aumenta em $2 \%$ a sua taxa de sobrevida. ${ }^{28}$

Após 30 a 32 semanas o Doppler de artéria umbilical é o mais importante indicador de dano fetal e é geralmente aceito que um fluxo diastólico ausente ou reverso seja uma indicação para antecipação do parto. ${ }^{6,28}$

Com relação à corticoterapia, ela deve ser realizada antes de 34 semanas nestes fetos, visando à diminuição das complicações neonatais e deve ser dada preferência à betametasona.

As figuras de 2 a 4 demonstram um algoritmo 


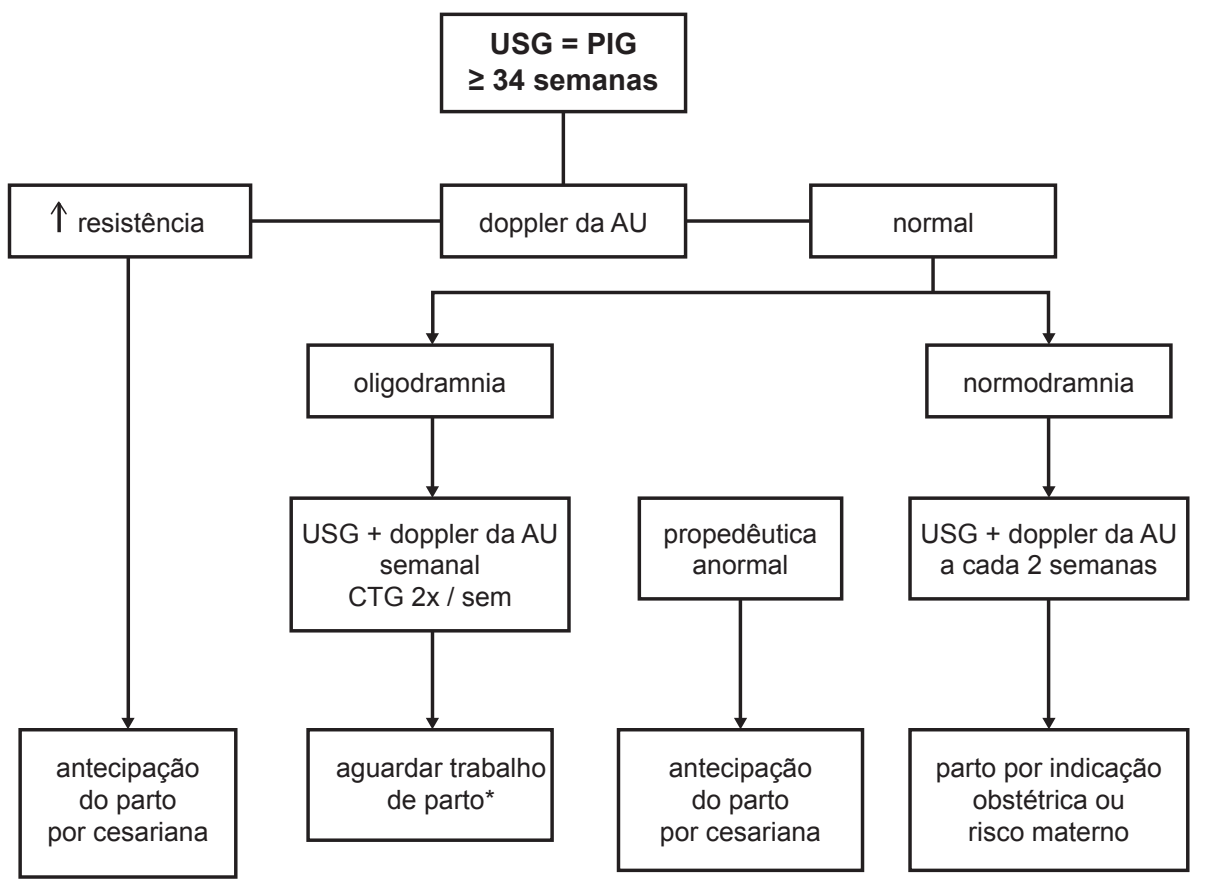

* Não ultrapassar a 39a semana

Figura 2: Condução dos fetos com restrição de crescimento com trinta e quatro semanas de gravidez ou mais.

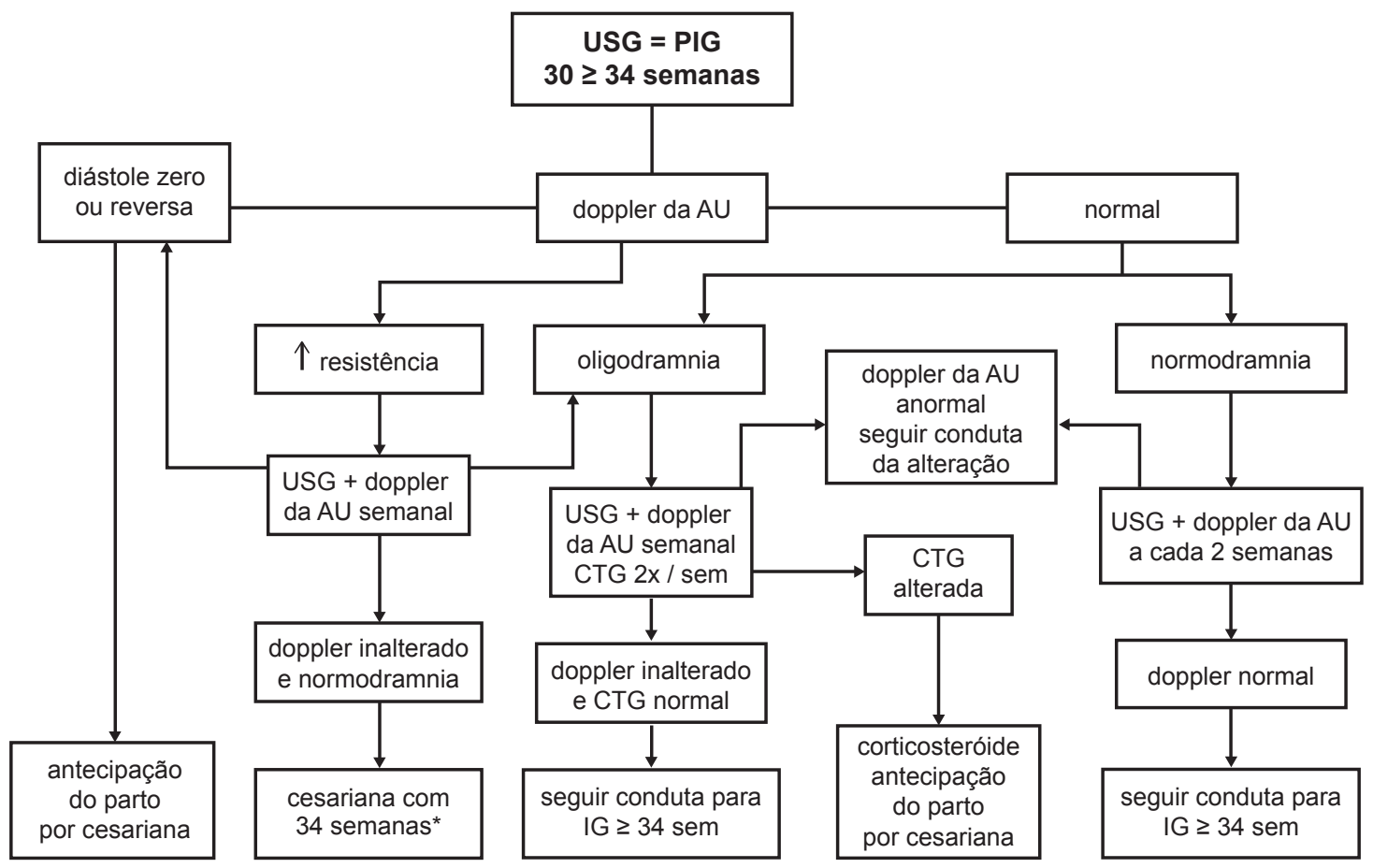

Figura 3: Condução dos fetos com restrição de crescimento entre trinta e trinta e quatro semanas de gravidez. 


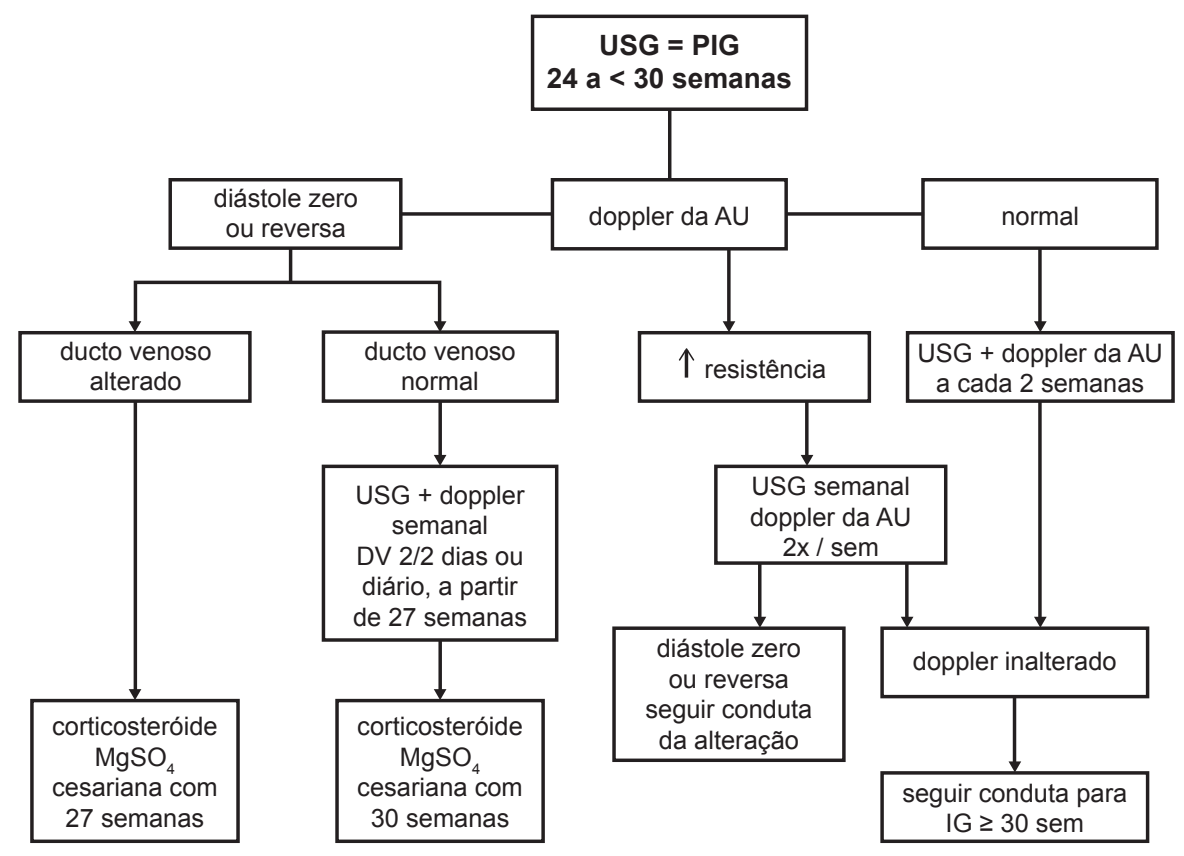

Figura 4: Condução dos fetos com restrição de crescimento entre 24 e 30 semanas de gravidez.

com a proposta de condução dos fetos com diagnóstico de restrição do crescimento intrauterino, com base na idade gestacional.

\section{Referências}

1. Kramer MS, Olivier M, McLean FH, Willis DM, Usher $\mathrm{RH}$. Impact of intrauterine growth retardation and body proportionality on fetal and neonatal outcome. Pediatrics. 1990;86(5):707-13.

2. Barker DJ. Adult consequences of fetal growth restriction. Clin Obstet Gynecol. 2006;49(2):270-83.

3. Lindqvist PG, Molin J. Does antenatal identification of small-for-gestational age fetuses significantly improve their outcome? Ultrasound Obstet Gynecol. 2005;25(3):258-64.

4. Lubchenco LO, Hansman C, Dressler M, Boyd E. Intrauterine growth as estimated from liveborn birth-weight data at 24 to 42 weeks of gestation. Pediatrics. 1963;32:793-800. [PMID: 14075621]

5. Sá RAM, Oliveira CA, Peixoto Filho FM, Lopes LM. Predição e prevenção com crescimento intrauterino restrito. Femina. 2009;57(9):311-14

6. Marsál K. Obstetric management of intrauterine growth restriction. Best Pract Res Clin Obstet Gynaecol. 2009;23(6):857-70

7. Montenegro CAB, Rezende J. Filho. Rezende Obstetrícia. 12. ed. Rio de Janeiro: Guanabara Koogan, 2013. 1200p
8. Figueras F, Gardosi, J. Intrauterine growth restriction: new concepts in antenatal surveillance, diagnosis and management. Am J Obstet Gynecol. 2011;204(4):288-300.

9. Villar J, Carroli G, Wojdyla D, Abalos E, Giordano $\mathrm{D}$, Ba'aqeel $\mathrm{H}$, et al. Preeclampsia, gestational hypertension and intrauterine growth restriction, related or independent conditions? Am J Obstet Gynecol. 2006;194(4):921-31.

10. Weeks JW, Asrat T, Morgan MA, Nageotte M, Thomas SJ, Freeman RK. Antepartum surveillance for a history of stillbirth: when to begin? Am J Obstet Gynecol. 1995;172(2 Pt 1):486-92.

11. Gardosi J, Francis A. Adverse pregnancy outcome and association with small for gestational age by customised and population based birthweight percentiles. Am J. Obstet Gynecol. 2009;201(1):28.e1-8.

12. Hollier LM, McIntire DD, Leveno KJ. Outcome of twin pregnancies according to intrapair birth weight differences. Obstet Gynecol. 1999;94(6):1006-10.

13. Gainer J, Alexander J, McIntire D, Leveno K: Fetal growth velocity in women who develop superimposed preeclampsia. Am J Obstet Gynecol. 2004:191(6):S170

14. Cunningham FG, Leveno KJ, Bloom SL, Hauth JC, Rouse DJ. Fetal Growth disorders. In: Williams Obstetrics 23 $3^{\text {rd }}$ edition 2010. Mac-Graw Hill.

15. Dugoff L, Hobbins JC, Malone FD, Porter TF, Luthy $\mathrm{D}$, Comstock $\mathrm{CH}$, et al. First-trimester maternal serum PAPP-A and free-beta subunit human 
chorionic gonadotropin concentrations and nuchal translucency are associated with obstetric complications: a population-based screening study (the FASTER Trial). Am J Obstet Gynecol. 2004:191:1446-51.

16. Smith GC, Stenhouse EJ, Crossley JA, Aitken DA, Cameron AD, Connor JM. Early pregnancy levels of pregnancy-associated plasma protein a and the risk of intrauterine growth restriction, premature birth, preeclampsia, and stillbirth. J Clin Endocrinol Metab. 2002;87(4):1762-7.

17. Killam WP, Miller RC, Seeds JW. Extremely high maternal serum alpha-fetoprotein levels at secondtrimester screening. Obstet Gynecol. 1991;78(2):257-61.

18. Lepage N, Chitayat D, Kingdom J, Huang T. Association between second-trimester isolated high maternal serum human chorionic gonadotropin levels and obstetric complications in singleton and twin pregnancies. Am J Obstet Gynecol. 2003;188(5):1354-9.

19. Aquilina J, Thompson O, Thilaganathan B, Harrington K. Improved early prediction of pre-eclampsia by combining second-trimester maternal serum inhibin-A and uterine artery Doppler. Ultrasound Obstet Gynecol. 2001;17(6):477-84.

20. Martin AM, Bindra R, Curcio P, Cicero S, Nicolaides $\mathrm{KH}$. Screening for pre-eclampsia and fetal growth restriction by uterine artery Doppler at 11-14 weeks of gestation. Ultrasound Obstet Gynecol. 2001;18(6):583-6.

21. Foidart JM, Munaut C, Chantraine F, Akolekar R, Nicolaides KH. Maternal plasma soluble endoglin at 11-13 weeks' gestation in pre-eclampsia. Ultrasound Obstet Gynecol. 2010;35(6):680-7.

22. Royal College of Obstetricians and Gynaecologists. The investigation and management of the small-forgestational age fetus. RCOG Green Top Guideline no. 31. London; Nov 2002. Available at: www.rcog.org.uk/ files/rcogcorp/loadedfiles/GT31SmallGestationalAge Fetus.pdf. Accessed Nov. 12, 2010.

23. Chang TC, Robson SC, Boys RJ, Spencer JA. Prediction of the small for gestational age infant: which ultrasonic measurement is best? Obstet Gynecol. 1992;80(6):1030-8.

24. Crispi F, Domínguez C, Llurba E, Martin-Gallán P, Cabero L, Gratacós E. Placental angiogenic growth factors and uterine artery Doppler findings for characterization of different subsets in preeclampsia and in isolated intrauterine growth restriction. Am J Obstet Gynecol. 2006;195(1):201-7.
25. Stampalija T, Alfirevic Z, Gyte G. Cochrane Reviews' summaries and their relevance for imaging: Doppler in obstetrics. Ultrasound Obstet Gynecol, 2010;36:779-80. http://dx.doi.org/10.1002/uog.8863.

26. Mari G, Deter RL. Middle cerebral artery flow velocity waveforms in normal and small-for-gestational-age fetuses. Am J Obstet Gynecol. 1992;166(4):1262-70.

27. Ferrazzi E, Bozzo M, Rigano S, Bellotti M, Pardi G, Battaglia F, et al. Temporal sequence of abnormal Doppler changes in the peripheral and central circulatory systems of the severely growth-restricted fetus. Ultrasound Obstet Gynecol. 2002;19(2):140-6.

28. Baschat AA. Cosmi E. Bilardo CM. Wolf H. Berg C. Rigano S, et al. Predictors of neonatal outcome in early-onset placental dysfunction. Obstet Gynecol. 2007;109(2 Pt 1):253-61.

29. Baschat AA. Neurodevelopment following fetal growth restriction and its relationship with antepartum parameters of placental disfunction. Ultrasound Obstet Gynecol. 2011;37(5):501-14.

30. Hecher K, Bilardo CM, Stigter RH, Ville Y, Hackelöer BJ, Kok HJ, et al. Monitoring of fetuses with intrauterine growth restriction: a longitudinal study. Ultrasound Obstet Gynecol. 2001;18(6):564-70

\section{Dailson D. S. Pereira}

Unidade Docente Assistencial de Obstetrícia. Hospital Universitário Pedro Ernesto. Faculdade de Ciências Médicas.Universidade do Estado do Rio de Janeiro. Rio de Janeiro, RJ, Brasil.

\section{Alessandra L. C. Magalhães}

Unidade Docente Assistencial de Obstetrícia. Hospital Universitário Pedro Ernesto. Universidade do Estado do Rio de Janeiro. Rio de Janeiro, RJ, Brasil.

\section{Nilson R. de Jesús}

Disciplina de Obstetrícia. Departamento Ginecologia e Obstetrícia. Faculdade de Ciências Médicas. Universidade do Estado do Rio de Janeiro. Rio de Janeiro, RJ, Brasil.

\section{Alexandre J. B. Trajano}

Departamento de Ginecologia e Obstetrícia. Faculdade de Ciências Médicas. Universidade do Estado do Rio de Janeiro. Rio de Janeiro, RJ, Brasil.

Departamento de Ginecologia e Obstetrícia. Faculdade de Medicina. Universidade Unigranrio. Rio de Janeiro, RJ, Brasil 